\title{
Ampliar la base de participación: el programa de tenis para niños de la LTA
}

\author{
Sam Richardson y Merlin van de Braam
}

\section{RESUMEN}

Este artículo comenta el Programa de Tenis para Niños de la LTA, que se creó tras la victoria de Gran Bretaña en Copa Davis en 2015. Desde entonces, la iniciativa ha logrado que 35.000 nuevos niños se inicien en el tenis y 15.000 continúen jugando. Este artículo comenta el enfoque de la formación de entrenadores, planes para clases, y también considera estrategias posibles para crear una iniciativa similar, y que más gente juegue más frecuentemente.
Palabras clave: Participación, iniciativa, Tenis para Niños (Tennis for Kids), Tenis para participantes

Recibido: 30 Ene 2016

Aceptado: 20 Jun 2017

Autor correspondiente: Sam

Richardson, LTA: The official

home of tennis for Britain.

Correo electrónico:

sam.richardson@lta.org.uk

\section{INTRODUCCIÓN}

En el centro del plan estratégico de la LTA está su misión: "Hacer que más gente juegue al tenis más frecuentemente". Con muchos deportes en declinación en el RU (Sport England, 2017), es necesario contar con entes gobernantes que demuestren los beneficios de cada deporte e implementen iniciativas para capturar tales beneficios con miras a aumentar la base de participación.

Las recientes investigaciones destacan los muchos beneficios para la salud asociados con el tenis. Específicamente, quienes eligen jugar tenis parecen lograr un mejor estado aeróbico, menor porcentaje de grasa corporal, un perfil lípido más favorable, menor riesgo de enfermedad cardiovascular y mejor salud ósea (Pluim y cols., 2007). Las investigaciones más recientes también sugieren que los tenistas viven más, gracias a los beneficios para la salud asociados.

Las investigaciones realizadas por la LTA indican que para lograr participantes para toda la vida, es clave iniciar el tenis desde temprana edad, preferiblemente antes de los 10 años, para aumentar significativamente la probabilidad de participación de por vida. Muy pocos adultos aprenden a jugar tenis desde cero, por lo tanto, el tenis se debe aprender en una edad temprana para tener posibilidades de mantener o recuperar a estos adultos más tarde (LTA, 2016). Adquiriendo las habilidades del tenis durante la juventud, se crean las bases para permanecer, o retornar al juego en cualquier momento. Ciertamente, las ventanas críticas para el desarrollo de la coordinación ocurren durante la niñez (SackeyAddo, Perez y
Crespo, 2016), lo cual refuerza la necesidad de que los niños comiencen a jugar al tenis (y otros deportes) a temprana edad.

\section{LA CAMPAÑA TENIS PARA NIÑOS}

La victoria de Gran Bretaña en Copa Davis en 2015 creó una oportunidad para inspirar a la siguiente generación de jugadores en el RU.

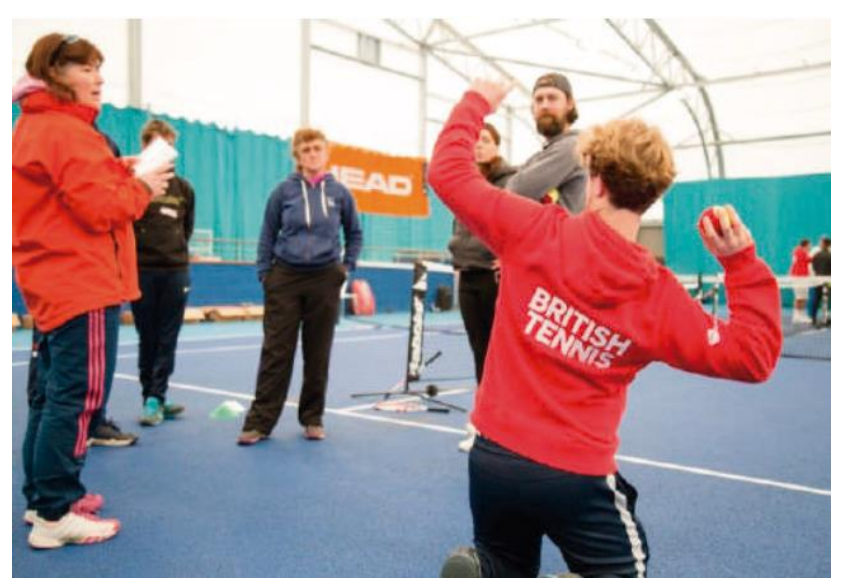

Imagen 1. Los entrenadores probando ejercicios en el curso de seis semanas, parte de los días de entrenamiento oficial.

La campaña Tenis para Niños se creó a partir de esta oportunidad. Dicha campaña fue una iniciativa de participación 
de gran escala para obtener 10.000 nuevos niños que jueguen al tenis por primera vez; y así diseñar y construir un legado duradero para la Copa Davis. Estos 10.000 niños, nuevos en el tenis, recibieron seis horas de entrenamiento gratuito en un curso de seis semanas llevado a cabo por entrenadores certificados. Los participantes, cuyas edades oscilaban entre los 5 y 8 años, recibían una raqueta gratis al completar el curso.

\section{LA CAMPAÑA TENIS PARA NIÑOS: PASOS CLAVE}

1. Solicitud de entrenadores: Todos los entrenadores acreditados de la LTA fueron invitados a solicitar ser parte de la campaña Tenis para Niños enviando un video explicando por qué querían participar. Más de 900 entrenadores lo solicitaron en el primer año, 875 asistieron a uno de los 16 días de entrenamiento para entrenadores llevado a cabo en el RU.

2. Formación de entrenadores: Los entrenadores debieron asistir a un taller de capacitación de cuatro horas al día que incluía semanas de planes para clases en cancha, cómo manejar las reservas para el curso y cómo promocionar estos cursos a nivel local. Se reclutó a un equipo de mentores para inspirar y compartir su conocimiento experto durante los días de entrenamiento, entre ellos estaban el capitán de Copa Davis, Leon Smith, los ex número uno británicos Annabel Croft, Anne Keothavong y Greg Rusedski. Los días de capacitación fueron increiblemente exitosos, con verdadero sentido de energía y entusiasmo, sumado a un propósito colectivo de aprovechar el momento inmediatamente después de la Copa Davis.

3. Apoyo de los entrenadores: Los entrenadores, trabajando con sus centros, crearon cursos en cualquier momento del año entre abril y septiembre para organizar las seis semanas de clases. Los cursos se publicaron y las reservas, se concretaban por medio de ClubSpark - un programa de reservas en línea. Durante los días de entrenamiento, los entrenadores fueron instruidos fuera de cancha y ayudados con sus necesidades, entre los cuales se incluía cómo hacer reservas en línea. El hecho de contar con una página de internet para las reservas permitió que la LTA pudiera, de manera efectiva, promocionar y comercializar la campaña con un evento nacional, reuniendo a los padres en un lugar en línea.

4. Conversión: Los entrenadores y los centros, todos trabajaron juntos para asegurar que los padres reciban un incentivo y quieran continuar jugando al tenis después del curso de seis semanas. Entre otros, se ofreció una membrecía a mitad de precio, o un porcentaje de descuentos sobre el programa estándar, que se estaba llevando a cabo en el lugar.

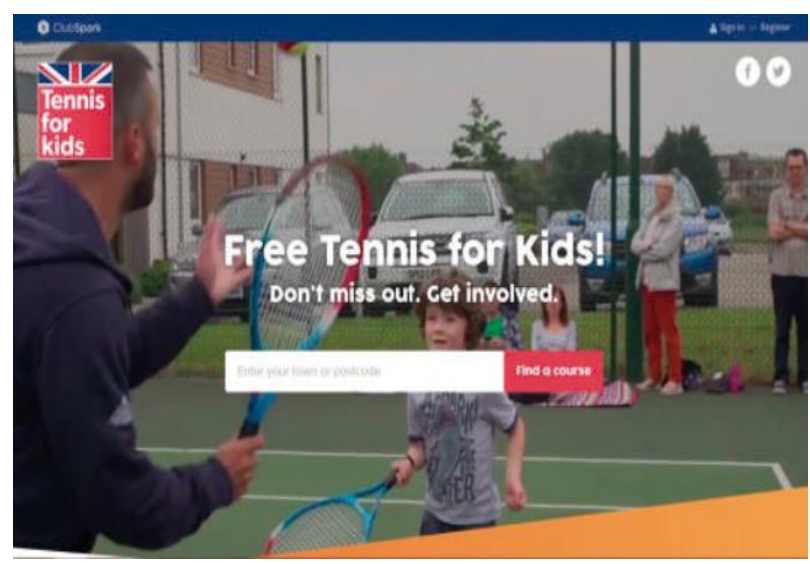

Imagen 2. La página de reservas en internet para que los padres encuentren sesiones locales de Tenis para Niños.

\section{LOS PLANES DE CLASES DE 6 SEMANAS}

Un panel experimentado de entrenadores desarrolló un conjunto de planes para las lecciones. El propósito de tales sesiones era desarrollar sólidos fundamentos atléticos y tenísticos, incluyendo las habilidades de "enviar y recibir", lanzar, agarrar y el desarrollo general de la coordinación. El objetivo del curso era guiar a los niños iniciantes para que puedan saca, pelotear y jugar puntos al final de las seis semanas. Dentro de las clases, se enfatizó la organización de grandes grupos, para que las sesiones tengan la máxima participación y "se parezcan al tenis".

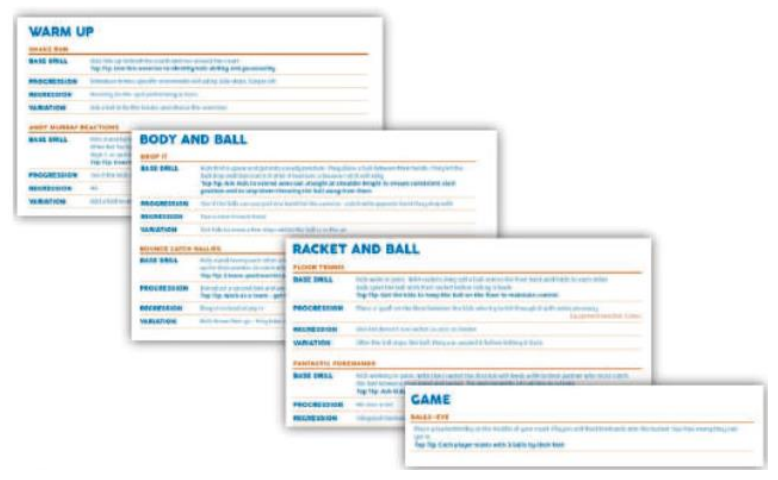

Imagen 3. La estructura del plan de lecciones para el curso de seis semanas.

\section{RESULTADOS DE LA CAMPAÑA}

En el año 1, el objetivo fue reclutar a 10.000 niños y niñas y mantener al 10\%. Esta expectativa fue excedida, 13.250 niños reservaron un curso y $43 \%$ se inscribió en un programa de entrenamiento después del curso, o se asoció a un club. Como resultado del enorme éxito en 2016, la LTA se comprometió con la campaña para 2017 y duplicó el objetivo con 20.000 niños recibiendo un curso gratuito durante 6 semanas y una raqueta también gratis. Desde la perspectiva promocional, tras su inicio nacional, "TennisForKids" estaba $7^{\circ}$ en twitter, 2 
semanas después logró las 20.000 reservas esperadas. En el momento de escribir este artículo, 22.200 niños han reservado un curso y otros miles están en lista de espera.

Para el año 2 el objetivo es aumentar la retención (hasta el 50\%) después de los cursos. Para esto, los entrenadores y los clubes deben trabajar juntos y proporcionar una propuesta sólida: o un programa de entrenamiento, o bien una membrecía. Para atraer a los padres de los niños inscriptos, la LTA proporcionó paquetes de apoyo por medio de Tennis Xpress (raquetas para adultos y pelotas verdes) y casi 500 entrenadores, lo que fue muy bien recibido tanto por éstos como por los padres.

\section{IMPLEMENTACIÓN DE LA CAMPAÑA TENIS PARA NIÑOS CON LIMITADOS RECURSOS}

Tenis para Niños contiene diversos elementos clave que ayudan a asegurar el éxito del programa. Sin embargo, uno o más de estos elementos se pueden reducir o hacer más factible financieramente para los países con limitados recursos. Algunas recomendaciones se mencionan a continuación:

- Las sedes pueden proporcionar la raqueta y el material gratuitamente, en lugar de la asociación Nacional - esto creará un vínculo y potencialmente aumentará la conversión después del curso, reduciendo en gran medida los costos del ente gobernante.

- En vez de tener un programa de búsqueda centralizado en línea, los centros podrán tomar reservas telefónicas y eliminar los costos de la construcción de una plataforma en línea. La página de internet de la Asociación Nacional debe enlistar los centros que participan y proporcionar los datos de contacto.

- Comenzando gradualmente, con 10 clubes, 100 nuevos niños pueden comenzar a jugar al tenis. Una vez conocidas las altas tasas de conversión, más centros adoptarán la iniciativa, probablemente, para el año 2

\section{CONCLUSIÓN}

Tenis para Niños fue una iniciativa dirigida a la bases de la participación, capitalizando el éxito logrado en la cúspide del juego. Por medio de una colaboración estrecha con los entrenadores, una nueva generación de niños se inició en el tenis bajo la mirada experta de entrenadores calificados. Es de esperar que la campaña continúe iniciando a más niños cada año, para asegurar así una participación de por vida en el juego de tenis.

\section{REFERENCIAS}

Lawn Tennis Association (2016). Insight presentation. London, UK.

Pluim, B. et al. (2007). Health benefits of tennis. Récupéré le 11 juillet 2017 à l'adresse http://www.tennisplayandstay.com/ media/133921/133921.pdf. https://doi.org/10.1136/bjsm.2006.034967

Sackey-Addo, R., Perez, J. et Crespo, M. (2016). Fundamental motor skills for 10 and $12 \&$ under tennis players. ITF Coaching \& Sport Science Review, 69, 6-9. https://doi.org/10.52383/itfcoaching.v24i69.185

Sport England (2017). The Active People Survey. Récupéré le 8 juillet 2017 à l'adresse https://www.sportengland.org/research/ aboutour-research/active-people-survey/

CONTENIDO ITF ACADEMY RECOMENDADO (HAZ CLICK ABAJO) $\mathbb{T T F}^{\circ}$ Academy

Derechos de Autor (c) 2017 Sam Richardson y Merlin van de Braam.

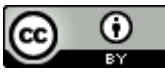

Este texto está protegido por una licencia CreativeCommons 4.0.

Usted es libre para Compartir -copiar y redistribuir el material en cualquier medio o formato- y Adaptar el documento -remezclar, transformar y crear a partir del material- para cualquier propósito, incluso para fines comerciales, siempre que cumpla la condición de:

Atribución: Usted debe dar crédito a la obra original de manera adecuada, proporcionar un enlace a la licencia, e indicar si se han realizado cambios. Puede hacerlo en cualquier forma razonable, pero no de forma tal que sugiera que tiene el apoyo del licenciante o lo recibe por el uso que hace de la obra.

$\underline{\text { Resumendelicencia - Textocompletodelalicencia }}$ 